\title{
Metalengua, metalenguaje, metadiscurso, algunos valores y usos aplicados a la enseñanza-aprendizaje de lenguas extranjeras Terencia Inés Silva Rojas Universidad de Vigo
}

The use of metalinguistic functions in the teaching of foreign languages is not restricted to employing raditional or decidedly explicit methods, as can be demonstrated through a series of empirical exercises as well as through a more exacting analysis of the matter. Nor is their use exclusive to the teacher. Language's potential for becoming its own object is a reality intrinsic not only to everyday communication but also to the communicative exchange proper to the learning/teaching of foreign languages. In this article three key notions are presented: 'metalangue', metalanguage and metadiscourse. These notions are born of the exercise of metalinguistic function and the differentiation between language as a descriptive instrument and language as an object, the aim being to examine their applications to the field of learning/teaching foreign languages.

Aunque su origen se encuentra en la lógica formal y matemática (Russel, Tarski y Whitehead) que diferencia el lenguaje objeto del metalenguaje, las nociones de metalenguaje y metalengua, tienen su difusión en la lingüística contemporánea gracias a las teorías de Jakobson y Hjelmslev.

Para Jakobson (1963) la función metalingüística constituye una de las seis funciones del lenguaje, formando parte de las tres, junto con la fática y la poética que añade a las otras tres establecidas por Bühler. En la función metalingüística, siempre presente en la comunicación verbal, el locutor toma el código lingüístico como objeto de su discurso para verificar si tanto él como el destinatario utilizan el mismo código. Esta verificación se realiza a través de diversos procedimientos discursivos como la simple repetición, la sinonimia, las locuciones, las paráfrasis, la traducción a otra lengua o incluso, a otro sistema de signos. Para 
Jakobson la interpretación metalingüística de los mensajes juega un papel esencial para el funcionamiento de una lengua natural así como para todo proceso de aprendizaje del lenguaje.

En su tipología sobre el lenguaje, Hjelmslev (1968) diferencia entre lenguas conformes, cuando los dos niveles que constituyen sus signos poseen exactamente la misma organización formal, como en el caso de los sistemas propios de los matemáticos y las lenguas no conformes, entre las que encontramos las lenguas denotativas cuando ninguno de los dos niveles es por sí mismo un lenguaje como en el caso de las lenguas naturales, las connotativas en las que el plano de la expresión es un lenguaje como en el caso de la literatura y la metalengua en la que el plano del contenido es en sí mismo un lenguaje, como en el caso de la lingüística.

Aunque la mayor parte de autores no distingue entre metalengua y metalenguaje dando a ambos la misma acepción general de lenguaje o lengua sobre el lenguaje, Besse (1. 1980) hace una distinción útil e interesante, incorporando también la noción de metadiscurso. La metalengua se situaría, según este autor, a nivel de la lengua natural y estaría constituida por los recursos léxicos, sintáxicos y pragmáticos de ésta que permitan decir algo sobre la lengua. El metalenguaje en cambio, estaría compuesto por un modelo conceptual cuyo objeto sería describir o simular el funcionamiento de una lengua natural o del lenguaje en general. El metadiscurso finalmente sería todo discurso que actualice un metalenguaje o una metalengua.

Para Borillo (1985) el metadiscurso es también fruto de la actualización de la metalengua y evoca fundamentalmente el acto de enunciación que le da origen. Posee un carácter reflexivo: es a la vez discurso y glosa sobre el discurso que lo origina. En este sentido Borilllo menciona tres rasgos que definen esta función de glosa:

- Hace referencia al discurso para explicar el código. Es el caso del niño en situación de aprendizaje o de situaciones de comunicación en las que se precisa, define, explica el sentido de lo que se quiere decir.

- Se hace referencia al discurso como un hecho enunciativo. En este caso cabe distinguir los enunciados que aseguran la eficacia comunicativa de ora categoría de enunciados caracterizados por 
el uso de performativos y expositivos cuya función es la de producir y orientar el discurso que contribuyen a constituir.

- Hace referencia al discurso desde la perspectiva de su propia construcción, a través de sus marcas de estructuración que operan sobre tres planos. la progresión lógica, la disposición y la argumentación.

En este trabajo vamos a presentar las principales aplicaciones de estas tres nociones sin otra intención que la de aclarar su utilización en el campo de la enseñanza-aprendizaje de lenguas extranjeras.

\section{1) Conocimiento explícito y conocimiento implícito}

Se trata de una dicotomía clásica resultado de la oposición entre una enseñanza basada en la explicación sobre el funcionamiento de la lengua enseñada, asociada a un conocimiento explícito que supondría la utilización de un discurso y actividades metalingüísticas en la clase, y la ausencia de tales explicaciones, y por consecuencia de elementos metalingüisticos, asociada a un conocimiento implícito. Esta oposición es una variable más amplia de otra dicotomía entre gramática, explícita y gramática implícita.

Pero la clase de lenguas extranjeras es un lugar en el que las prácticas metalingüísticas son casi constantes. Después de observar una muestra de clases de francés lengua extranjera, Besse (2.1980) comprueba que la cantidad de frases no metalingüísticas es considerablemente más reducida que el de las metalingüísticas, empleándose únicamente en tres circunstancias:

- frases cuya finalidad es la de formular actos en relación al funcionamiento de la clase como: buenos días, hasta mañana, etc.

- comentarios sobre personajes, imágenes o situaciones visualizadas

- paráfrasis discursivas inventadas por los estudiantes en situaciones y con personajes figurados.

Pero además de este carácter cuantitativo, la naturaleza misma de la clase de lenguas extranjeras es casi constantemente metalingüística 
en la medida en la cual los actos de lengua que en ella se realizan son objeto de mención y no de uso, a diferencia de una situación real de comunicación en lengua materna (Coste 1985). Es en este sentido que Rey-Debove (1983) considera el aprendizaje de las lenguas extranjeras como un tipo de discurso cuyo único tema es el lenguaje. Los enunciados que en él se emiten, tanto orales como escritos, tienen el mismo valor que los ejemplos del diccionario, un valor de citación.

Según lo que acabamos de ver se puede afirmar con Besse (1980) que toda enseñanza-aprendizaje en contexto institucionalizado pone en práctica metadiscursos inscritos en una metalengua o un metalenguaje. Los únicos casos posibles y aún con reservas, en los que se pueda dar un aprendizaje de la lengua extranjera en el que se excluya los elementos metalingüísticos son: por un lado, los de un aprendizaje sin ningún tipo de escolarización, basado en el simple contacto y el intercambio comunicativo con los hablantes autóctonos y el de los niños de corta edad, por otro, que no han desarrollado todavía una consciencia lingüística de su lengua materna.

Por otro lado, conviene señalar que la oposición entre conocimiento explícito y conocimiento implícito simplifica y reduce la lengua objeto, al conocimiento que de ésta se pretende conseguir, confundiéndolo además con el medio o los medios (modelo teórico, prácticas lingüísticas o pedagógicas) que se emplean para obtener este conocimiento. Además de resultar insuficiente para explicar tanto los procesos cognitivos como la actividad lingüística puesta en práctica en los procesos de adquisición.

En todo caso más que de una dicotomía entre medios intuitivos o carentes de una formalización teórica y reglas explícitas de funcionamiento o un discurso científicamente elaborado, las clases de lenguas extranjeras parecen ser más bien, una realidad indisociable de prácticas metalingüísticas, tanto metalengua como metalenguaje, de prácticas lingüísticas y prácticas epilingüísticas, la actividad metalingüística no consciente (Bally 1980). Es necesario recordar el papel que juegan estas últimas a menudo relegadas a una situación secundaria, confrontado por trabajos empíricos que prueban su importancia en relación al aprendizaje de lenguas extranjeras (Krzeminska 1986). 


\section{2) Metalenlingüística y adquisición,}

También podemos enfocar la actividad metalingüística en oposición a los procesos de adquisición de una lengua que suponen la puesta en práctica de una interacción y una comunicación reales, frente a los procesos de aprendizaje que implican en cambio la construcción de representaciones mentales de las reglas lingüísticas y una actividad consciente y sujeta a control por parte del sujeto que aprende. (Krashen 1981). Para este autor únicamente la adquisición es fuente de una verdadera apropiación de la lengua, mientras que la reflexión gramatical sólo es capaz de dar cuenta de una parte reducida del funcionamiento de la lengua.

Muchos autores comparten la opinión de Krashen en lo que a la adquisición natural de la lengua, extranjera o materna se refiere; pero se apartan o matizan sus teorías reconociendo que la conceptualización metalingüística puede contribuir al proceso de adquisición en condiciones de aprendizaje escolar o institucionalizado. Coste (1985) recoge y señala varias hipótesis:

- Una reflexión metalingüística guiada puede impedir que la consciencia lingüística espontánea perturbe o retrase la adquisición natural de la lengua extranjera.

- Las descripciones metalingüísticas se refieren más al funcionamiento de la lengua objeto en su funcionamiento canónico que a los estados de interlengua. Por consiguiente, si se introducen demasiado pronto en la enseñanza corren el riesgo de no dar ningún resultado valorable, pero si se introducen en el momento adecuado pueden contribuir efectivamente a acelerar los procesos de maduración lingüística.

- Las descripciones adecuadas contribuyen a la economía de los procesos de adquisición.

- El trabajo metalingüístico explícito puede también ayudar de manera muy eficaz a desbloquear la fosilización y en general los procesos inacabados de evolución, etc. 


\section{3) Actividad metalingüística y enseñanza-aprendizaje a nivel avanzado}

En primer lugar tenemos que señalar que esta enseñanza no puede considerarse como la simple continuación de un aprendizaje anterior, sino que implica estrategias diferentes por parte del sujeto que aprende la lengua.

Un estudiante de nivel avanzado es capaz de poner en funcionamiento determinadas capacidades metalingüísticas y de establecer y objetivar un sistema de correspondencias interlingüísticas entre su lengua materna y la lengua que aprende, la lengua objeto, procedimientos que suponen un nivel de madurez lingüística que un estudiante que aún no ha interiorizado de manera sólida los mecanismos de funcionamiento de la lengua objeto, no es capaz de realizar. Pero además, tiene la necesidad, según señala Porquier (1993) de comparar el funcionamiento, de su interlengua y de la lengua objeto. Esta confrontación implica igualmente la posibilidad de desarrollar nuevas estrategias para acceder a la lengua objeto, en cuya elaboración, la reflexión metalingüística juega un papel esencial.

\section{4) Actividad metalingüística y culturas gramaticales}

Podemos señalar otro tipo de procedimiento metalingüístico que actúa sobre el aprendizaje de las lenguas exwanjeras, pero que no corresponde necesariamente ni a una explicación ni a una reflexión metalingüística o interlingüística, ni tampoco a mecanismos cognitivos de la adquisición. Se trata de representaciones sociales y culturales de naturaleza metalingüística sobre las que Beacco (1980), (1993) llama la atención, planteando que es importante tomar en cuenta, las fuentes en las cuales se originan las culturas gramaticales presentes en la enseñanzaaprendizaje de lenguas extranjeras.

Las descripciones de la lengua, nos dice, no son inocentes, al conrario, su elaboración hecha mano a conocimientos lingüísticos que necesitan de una congruencia sociolingǘstica para asegurar la coherencia de los soportes pedagógicos a utilizar en las prácticas de clase. Debemos preguntamos pues sobre los efectos que tienen las descripciones de la lengua sobre las interlenguas, sin olvidar que estas descripciones metalingüísticas se transmiten con técnicas de divulgación diferentes. 
En cuanto al alumno hay que diferenciar entre los dos aspectos de su competencia metalinguística: un rasgo mas bien pragmático, su saber-hacer, por un lado, y por otro, su herencia cultural de la que es necesario contar con descripciones objetivas.

Además existe lo que el autor llama "las presiones metalingüísticas", es decir, la demanda social de los usuarios. Aunque sus contextos lingüísticos y culturales están por determinar, nos menciona algunas variables que condicionan su existencia tales como:

- el peso de la tradición descriptiva de la lengua materna,

- el lugar ocupado por el lenguaje en los comportamientos culturales,

- el nivel de competencia en lengua extranjera del alumno,

- la edad de los alumnos,

- los estilos cognitivos individuales

- la proximidad cultural y estructural de las lenguas, etc.

Conviene insistir brevemente en la primera de las variables que acabamos de enumerar porque muestra la importancia que adquiere el metalenguaje empleado en la enseñanza-aprendizaje de la lengua materna para los mismos procesos en lengua o lenguas extranjeras, al actuar como el elemento mediador a través del cual se percibe e interpreta el funcionamiento de la lengua objeto.

\section{5) Metadiscurso y enseñanza-aprendizaje de lengua extranjeras}

Besse (1980) distingue dos tipos de metadiscurso: uno próximo a lo que él denomina actividades ordinarias del lenguaje que sólo requieren una cierta competencia de la lengua en la cual se inscribe y otro tipo especializado de metadiscurso que requiere de metalenguajes más o menos científicos. Entre ambos no hay solución de continuidad y se sitúan, por así decir, en polos opuestos.

Los primeros no presuponen el conocimiento de ningún metalenguaje. y su práctica didáctica sólo implica una enseñanza-aprendizaje parcial de la metalengua de la lengua objeto. Como procedimientos propios a este tipo de metadiscurso encontramos la autonimia y, sobre todo, la glosa metadiscursiva (sinonimias, circunlocuciones, paráfrasis diver- 
sas, etc.) que pueden desempeñar un papel fundamental en la enseñanza-aprendizaje de lenguas extranjeras. Formuladas por el profesor permiten explicar lo que todavía no conoce el alumno, empleando para esta labor signos que le son conocidos, lo que favorece la memorización de la nuevas adquisiciones. Formuladas por el alumno constituyen un medio de verificar sus hipótesis interpretativas sobre los hechos lingüísticos o no lingüísticos relativos a la lengua que aprende.

En cuanto a los segundos, Besse señala que por lo general los puntos de vista metalingüísticos del profesor y los alumnos nunca coinciden en una clase de lenguas extranjeras. Sin embargo, los metadiscursos lingüísticos no pueden evitarse a no ser que los alumnos no hayan aprendido ningún metalenguaje no sólo en relación a una lengua extranjera sino también a su propia lengua materna.

Finalmente y (antes de terminar,) queremos señalar que la actividad metalingüistica de descripción de una lengua extranjera no se identifica única y necesariamente con la actividad verbal escrita u oral; sino que puede recurrir, como de hecho ocurre, a otra clase de medios como la utilización de esquemas, de cuadros o de todo tipo de disposición gráfica, e incluso al auxilio de los recursos gestuales. Estamos pues en el terreno de la semiótica otra vertiente en el estudio de enseñanza-aprendizaje de lenguas extranjeras.

\section{BIBLIOGRAFIA}

BARTHES, R. 1964. "Eléments de sémiologie". Communications.4 . Paris.

BARTHES, R. 1967. Système de la mode. Paris: E. de Seuil.

BARTHES, R. 1968. Eléments de sémiologie. Paris: Gonthier.

BAILLY, D. 1980. "Synthèse de la dicussion sur connaissance implicite et connaissance explicite en langue maternelle". Encrages, numéro spécial: Acquisition d'une langue étrangère. Saint-Ienis, Université de Paris VIII.

BAILLY, D. 1992. "Le déclaratif et le procédural dans l'appropriation psycolingüistique par des francophones de la catégorie du genre en anglais et en allemand" en Acquisition et enseignement/apprentissage des langues. Actes du Colloque de Grenoble, mai 1991. Grenoble: Université Stendhal. 


\section{Terencia Inés Silva Rojas}

Metalengua, metalenguaje, metadiscurso, algunos valores y usos ...

Besse, H. 1. 1980. "Métalangages et apprentissage d'une langue étrangère". Langue française. 47. Paris.

Besse, H. 2. 1980 "Le discours métalinguistique de la classe". Encrages, numéro spécial: Acquisition d'une langue étrangère. Saint-Denis: Université de Paris VIII.

Besse, H. et PORQUIER, R. 1984. Grammaires et didactiques de langues. Paris: Hatier-CREIIF.

Beacco, J.C. et LEHMANN, D. 1980. "L'intervention didactique et les variables culturelles". Le Français dans le monde. 199. Paris.

Beacco, J.C. 1993. "Cultures grammaticales et demande métalingüistique". Etudes de lingüistique appliquée. 92. Paris.

Bourguignon, C. 1983. "Le métalangage un point de rencontre obligé entre enseignants de langue maternelle et de langue étrangère". le Français dans le monde. 177. Paris.

Bronckart, J.P. Szciner, G. 1990. "Description grammaticale et principes d'une didactique de la grammaire". Le français aujourd' hui. 89. Paris.

Chevalier, J.C. 1987. "Grammaire et enseignement des langues". Le Français dans le monde, numéro spécial : Vers un niveau 3. Paris.

Coste, I). 1984. "Les discours naturels de la classe". Le Français dans le monde. 183. Paris.

Coste, I). 1. 1985. "Sur quelques aspects des relations récentes entre grammaire et didactique du français langue étrangère". Langue française. 68. Paris.

Coste, I. 2. 1985. "Métalangages, activité métalinguistique et enseignement/apprentissage d'une langue étrangère". DRALAV. 32. Université de Paris VIII. Paris.

Cicurel, F. 1984. "La conquête du sens".Le Français dans le monde. 183. Paris.

Cicurel, F. 1985. Parole sur parole ou métalangage dans la classe de langue. Paris: CLE international.

Dabene, L. 1979. "La grammaire en langue maternelle et en langue étrangère". Etudes de lingüistique appliquée. 34. Paris.

Darot, M. 1983. "Quels outils pour l'analyse linguistique ou les préalables d'un enseignement de la grammaire". Le français dans le monde. 177. Paris.

Gaonac'h, I). 1987. Théorie d'apprentissage et acquisition d'une langue étrangère. Paris: Didier-Hatier.

Gaonac'h, D. 1990. “Acquisition et utilisation d'une langue étrangère. L'approche cognitive". Le français dans le monde. numéro spécial. Paris.

Germain, CL. 1993. Evolution de l'enseignement des langues: 5000 ans d' histoire. Paris: CLE international.

Hjelmslev, L. 1966. Le langage. Paris: Ed. de Minuit.

Hjelmslev, L. 1968. Prolégomènes à une théorie du langage. Paris: Ed. de Minuit. 
Jakobson, R. 1963. Essais de linguistique générale. Paris: Ed. de Minuit.

Krashen, S. 1978. "The Monitor Model for Second Language Acquisition". GRINGRAS, Second language acquisition and foreign language teaching. Arlington, Virginia.

Krashen, S. 1981. Second Language Acquisition and Second Language Teaching. Oxford: Pergamon Press.

Krashen, S. Terrell, D. 1983. The Natural Approach. Language Acquisition in the classroom. New York: Pergamon Press.

Krzeminska, W. 1986. "Les mécanismes des décisions métalinguistiques en langue étrangère" en Actes du Sème colloque international, Aix-en Provence, 1984, Acquisition d'une langue étrangère, Tome I, Université de Aix-en-Provence.

Klein, W. 1989. L'acquisition de langue étrangère. Paris: A. Colin.

Porquier, R. 1993. "Le statut des outils métalinguistiques dans l'apprentissage et l'enseignement au niveau avancé". Etudes de lingüistique appliquée. 92. Paris.

Rey-Debove, J. 1967. “Autonymie et métalangage”. Cahiers de lexicologie II. Paris.

Rey-Debove, J. 1972. "Le métalangage comme système de référence au signe". Le Français moderne. 3. Paris.

Rey-Debove, J. 1978. "Le métalangage" en Le Robert. Paris.

Rey-Debove, J. 1983. "Le métalangage dans la langue parlé”. Recherches sur le français parlé, 5. U. de Aix en Provence. Provence.

Rey-Debove, J. 1985. "Le métalangage en perspective”. DRLAV, 32. Paris.

Vives, R. 1989. "D'hier à aujourd'hui: la grammaire dans tous ses états". Le Français dans le monde. Et la grammaire... Paris.

Veronique, D. 1990. “A la rencontre de l'autre langue: réflexions sur les représentations dans l'apprentissage d'une langue étrangère". Le français dans le monde. Publics spécifiques et communication spécialisée. Paris. 